\title{
Genetic Algorithm with Virus Infection for Finding Approximate Solution
}

\author{
Takuya Inoue, Yoko Uwate and Yoshifumi Nishio \\ Tokushima University \\ 2-1 Minami-Josanjima, Tokushima 770-8506, JAPAN \\ Email: \{t-ionue, uwate, nishio\}@ee.tokushima-u.ac.jp
}

\begin{abstract}
Genetic Algorithm (GA) is modeling behavior of evolution in organic and known as one of method to solve Traveling Salesman Problem (TSP). However, GA obtains solution by overlaying generations because of being based on evolution in organic. Thus, it takes a long time to find approximate solution. While, Virus Theory of Evolution (VTE) can evolve by virus infection. VTE characteristic has sharing of information among same generation. If new algorithm is using both these characteristics of GA and VTE, convergence speed would be faster than GA. Thus, this study proposes Genetic Algorithm with Virus Infection (GAVI). GAVI algorithm is Virus Theory of Evolution (VTE) to be based on Genetic Algorithm (GA). We apply GAVI to TSP and confirm that GAVI obtains more effective result than GA.
\end{abstract}

\section{INTRODUCTION}

Traveling Salesman Problem (TSP) [1] is known as one of the combinatorial optimization problems. When Salesman tours all cities at once, TSP is the problem of finding minimum total between each city distance in route. Then $n$ is the number of cities in TSP, total route number increase at rate proportional to many of the factorial of $n$. For example, when the number of $n$ increases, the total route number explode in Table I. Therefore, exploring total route number needs amount of time in finding approximate solution. However we would like to obtain the approximate solution in TSP quickly. It is necessary to solve the TSP in other ways except exploring total routes. Finding approximate solution method of TSP is a variety of ways.

Genetic Algorithm (GA) [2], [3] is one of the popular method in variety of ways to solve the TSP and is studied by many researchers all over the world. GA is modeling behavior of evolution in organic. In genetic group, individuals with high evaluation tend to be many crossover. Thus, genetic group obtains high evaluation in average for repeating crossover. However, this method needs amount of time and convergence speed is slow. We would like to obtain approximate solution quickly. Thus, we need to propose the other method.

In this study, we propose Genetic Algorithm with Virus Infection (GAVI). One of the characteristics of the virus infection [4]-[6] conveys gene information among same generation at once. This characteristic seems to be useful for finding the approximate solution quickly. Therefore, we consider that the virus infection will help to solve the TSP. We carry out computer simulations for various parameter values and confirm that the proposed algorithm achieves better performance than the conventional GA.

TABLE I

THE NUMBER OF CITIES AND THE TOTAL ROUTE NUMBER

\begin{tabular}{|c|c|}
\hline number of cities & total route number \\
\hline 3 & 1 \\
4 & 3 \\
8 & $2.52 * 10^{3}$ \\
16 & $6.54 * 10^{11}$ \\
32 & $8.22 * 10^{33}$ \\
64 & $9.91 * 10^{86}$ \\
128 & $1.51 * 10^{213}$ \\
\hline
\end{tabular}

\section{Virus TheORY OF EVOLUTION}

Organic evolution is theory based on natural selection. In natural world, high fitness individuals organism survive, while low fitness individuals organism become extinct. Over the years, only higher fitness individuals survive. We call it Evolution. Thus, evolution need to overlay generations.

On the other hand, there is theory that Virus Theory of Evolution (VTE) [7]. This theory is based on the evolution by Lateral Gene TransferiLGT) [8] in Virus infection. LGT is uptake of the gene that occur between other individuals and among other species. Without evolution inherited from parent cell to child cell, genes can evolve. Low fitness individuals possibly evolve into high fitness individuals in just one generation by LGT in Virus infection. Thus, we assume using VTE algorithm leads the approximate solution in less time and VTE theory is efficient in TSP.

\section{Genetic Algorithm With ViRUs InfECtion}

Selection, Crossover and Mutation are the main functions of the GA. GAVI is a method of VTE algorithm in Virus infection to be based on GA. Flow chart of GAVI is shown in Fig. 1. New algorithm is Infection in Fig. $1 . t$ is the number of repeating times.

\section{Initialization}

Initialization is random route selection. Number of random route selection is $U$. 


\section{Evaluation}

Evaluation is defined by the following formula.

$$
f_{i}=\frac{1}{d_{i}}
$$

where $d_{i}$ is total distance of each route and $f_{i}$ is evaluation value. If $d_{i}$ is low, $f_{i}$ is high by this formula.

\section{Selection}

Route is selected with a probability of $p_{i}$. $p_{i}$ is defined by the following formula.

$$
p_{i}=\frac{f_{i}}{\sum_{i=1}^{n} f_{i}}
$$

where $n$ is the number of cities. Selection is the method of tends to be chosen high evaluation route. Low evaluation route is chosen a few. Selection works that high fitness individuals organism survive, while low fitness individuals organism become extinct.

\section{Fulfill crossover condition}

This section evaluates crossover condition. If parents is not fulfill crossover condition, crossover is not acted. When crossover condition is fulfill, crossover is acted.

\section{Crossover}

Crossover is to be mated the two routes. In this study, we apply sub tour exchange crossover. This way makes a search for sub tour of the both Parent A and Parent B in common. If it does not find sub tour in common, crossover algorithm is not acted. For example, between 1, 2, 5, 6 and 5, 1,6, 2 are sub tour in Fig. 2. 1, 2, 5, 6 and 5, 1, 6, 2 are differ in line, however these are same class. Sub tour in 1,2,5,6 can express $1,2,5,6$ and $6,5,2,1,5,1,6,2$ can express $5,1,6,2$ and $2,6,1,5$. Because two expressing are same about total route distance in Fig. 3. Thus, after crossover, four child exist.

\section{Mutation}

Mutation is to change the route with a certain probability in Fig. 5 and less likely to fall into local minimum.

\section{Infection}

We define elements of each route as Virus, elements select the best route in the obtained solution and part of each route is infected by this Virus at random. Number of element selects is fixed probability. For example, 3,5 is a virus and has infected the route of $6,1,3,5,2,4$ in Fig. 4. Infection part determines 1,4 in the route. The route replace to $3,51,4$. We call it Infection. Infection is incorporating partial optimum solution.

\section{One route reset in random}

If the obtained solution is same among number of $s$, one route in all routes is initialization in random. $O(t)$ is the obtained solution in number of $t$ times, while $O(t-s)$ is the obtained solution previous number of $s$. Thus, $O(t)=O(t-s)$ shows that the obtained solution is same among number of $s$. We assume that this is efficient to escape local minimum.

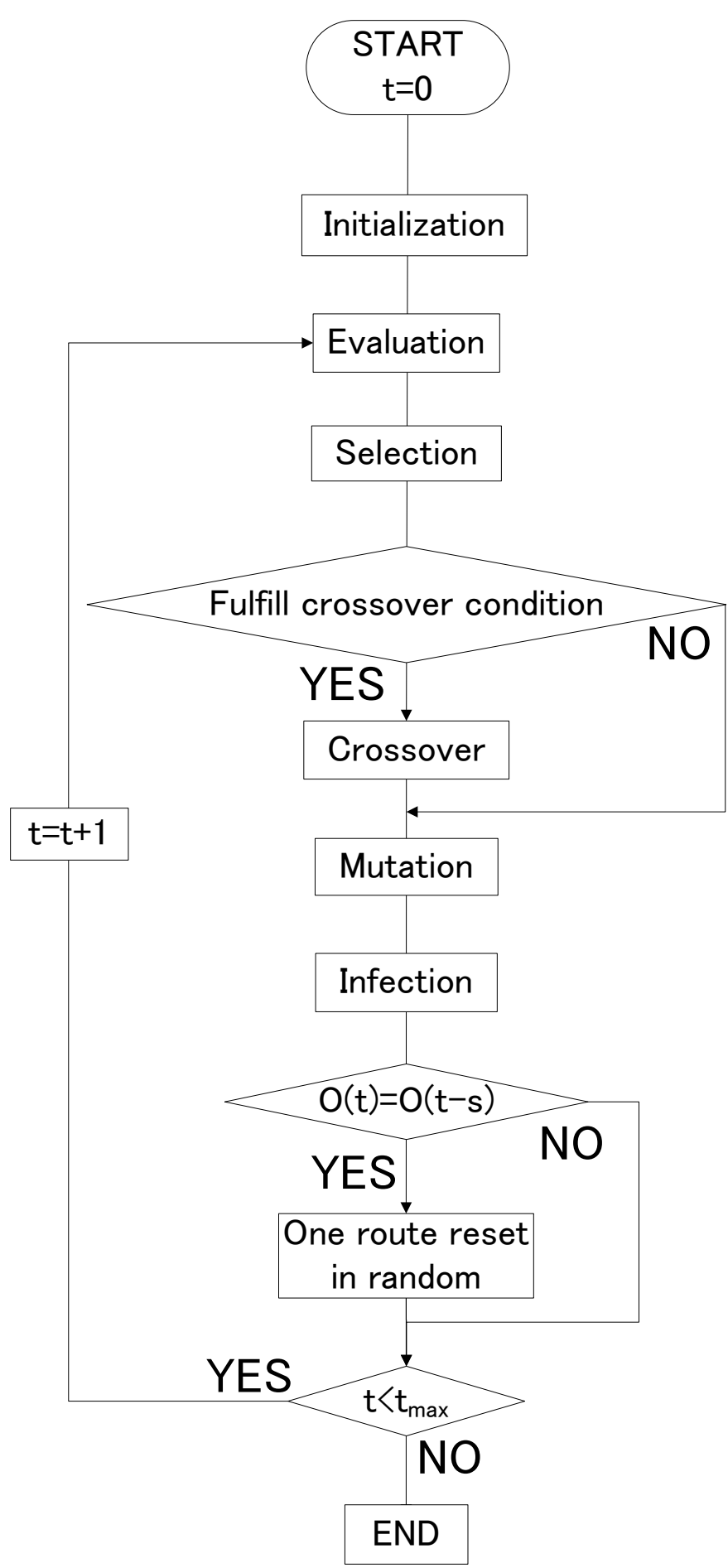

Fig. 1. Flow chart of GAVI 


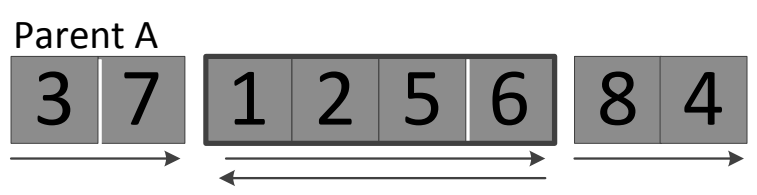

Parent B

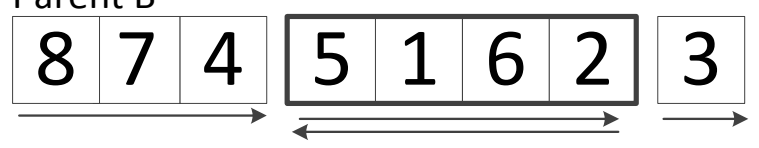

Child A

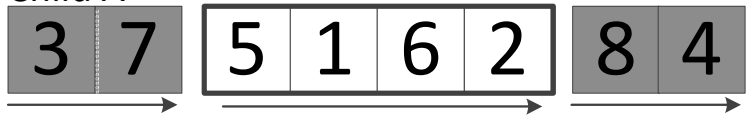

\section{Child B}

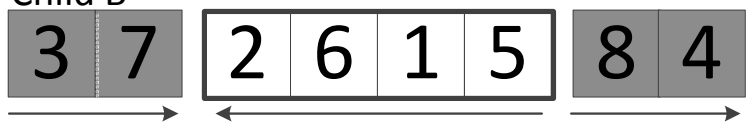

Child C

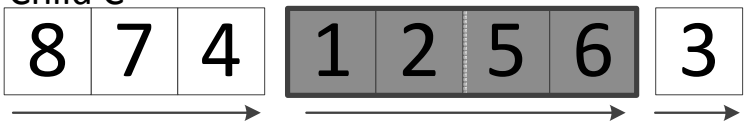

Child D

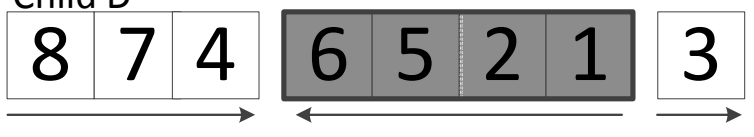

Fig. 2. The mechanism of Infection.

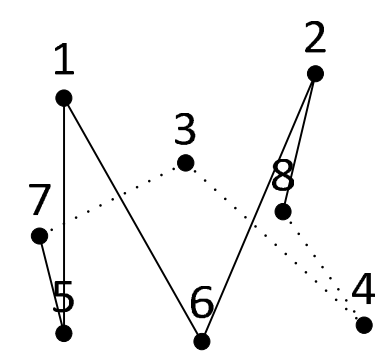

Child A

$(3,7,5,1,6,2,8,4)$

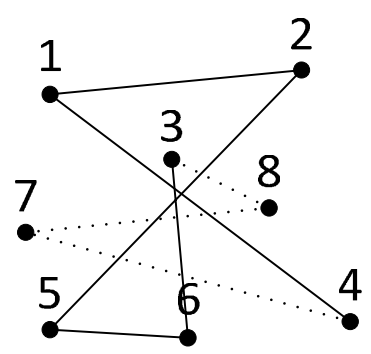

Child C

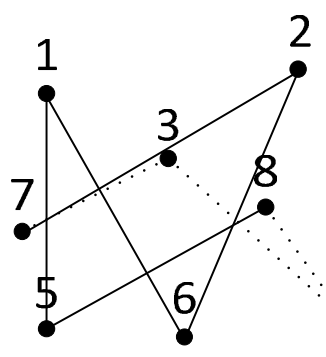

Child B $(3,7,2,6,1,5,8,4)$

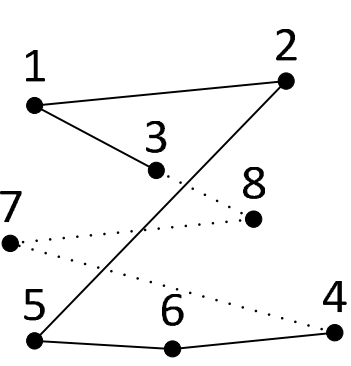

Child D

$(8,7,4,6,5,2,1,3)$
$(8,7,4,1,2,5,6,3)$

Fig. 3. The mechanism of Infection.

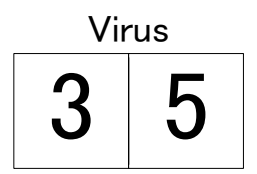

Choose a place to infect

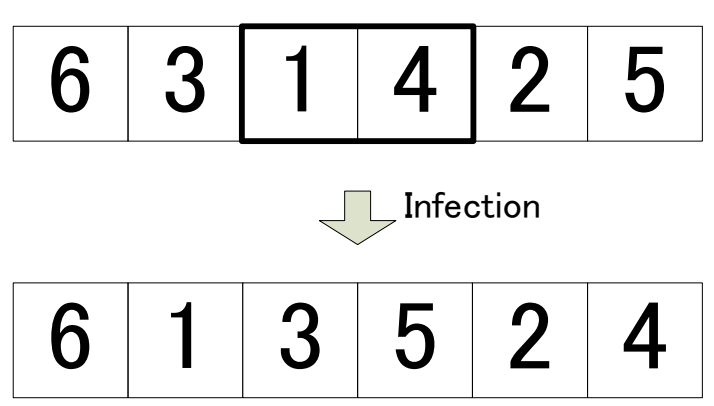

Fig. 4. The mechanism of Infection.
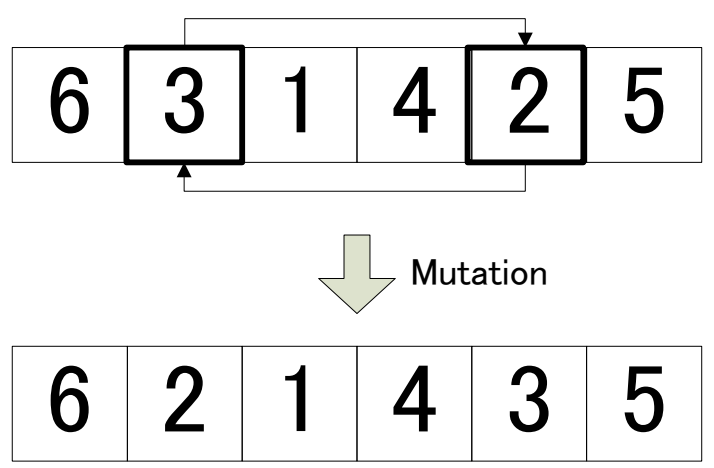

Fig. 5. The mechanism of Mutation.

\section{EXPERIMENTAL RESULTS}

In order to compare the performance of GAVI and GA, we apply GAVI and GA to find approximate solutions in TSP such as att48 (48 cities). In this study, the number of $t$ is 5000 times, the number of simulation is 10 times, $U$ is 1000 , number of route in and error rate is defined by the following formula.

$$
\text { Error rate }[\%]=\frac{(\text { obtain })-(\text { optimum })}{(\text { optimum })} \times 100
$$

where obtain shows obtained solution and optimum shows optimum solution. When obtain value approaches optimum value, Error rate is low. For example, when obtain value is equally optimum value, Error rate value is $0[\%]$. If Error rate value is 0[\%], we would obtain optimum solution. However, obtain is bad solution, error rate value is high. In Table II, III, Ave is the average, Max is the maximum value and Min is the minimum value in the results of simulation 10 times.

Table II shows result of GA and Table III shows result of GAVI. In GAVI, $s$ is fixed with 100 and Mutation rate is fixed with 0.02. GAVI can find a good solution by 
Infection from result of both Table II and Table III. When Infection rate is 0.01 , result of GAVI is the best. We assume that if Infection rate is high, escaping local minimum is difficult and diversity solution is loss. Thus, result of Infection rate $=0.1$ is the better than Infection rate $=$ 0.2 .

Figure 6 shows the error curve of GA and Fig. 7 shows the error curve of GAVI. The number of simulation is 1 time, Mutation rate is fixed with 0.02 about GA and GAVI, Infection rate is fixed with 0.10 about GAVI. In Figure 6 and 7, Convergence speed of GAVI is faster than GA. Thus, we assume that Infection algorithm is efficient at first.

TABLE II

THE RESULT FOR GA IN ATT48

\begin{tabular}{|c|c|ccc|}
\hline Algorithm & Mutation & \multicolumn{3}{|c|}{ Error rate[\%] } \\
\cline { 3 - 5 } type & rate & Max & Min & Ave \\
\hline & 0.02 & 6.486 & 0.597 & 2.878 \\
GA & 0.05 & 5.610 & 2.439 & 3.430 \\
& 0.10 & 4.621 & 1.280 & $\mathbf{2 . 0 9 6}$ \\
& 0.20 & 3.936 & 0.958 & 2.324 \\
\hline
\end{tabular}

TABLE III

THE RESULT FOR GAVI IN ATT48

\begin{tabular}{|c|c|ccc|}
\hline Algorithm & Infection & \multicolumn{3}{|c|}{ Error ate[\%] } \\
\cline { 3 - 5 } type & rate & Max & Min & Ave \\
\hline & 0.05 & 4.437 & 0.926 & 1.912 \\
GAVI & 0.10 & 2.835 & 0.530 & $\mathbf{1 . 1 9 5}$ \\
& 0.20 & 2.096 & 0.798 & 1.628 \\
\hline
\end{tabular}

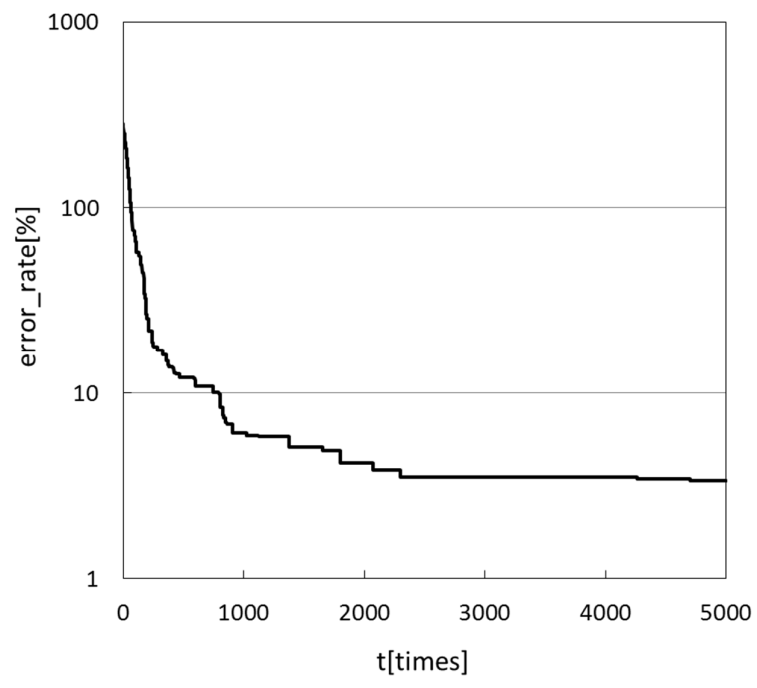

Fig. 6. error curve of GA.

\section{CONCLUSION}

We proposed GAVI for TSP and compared the performance of GAVI and GA to lead approximate solutions. From the

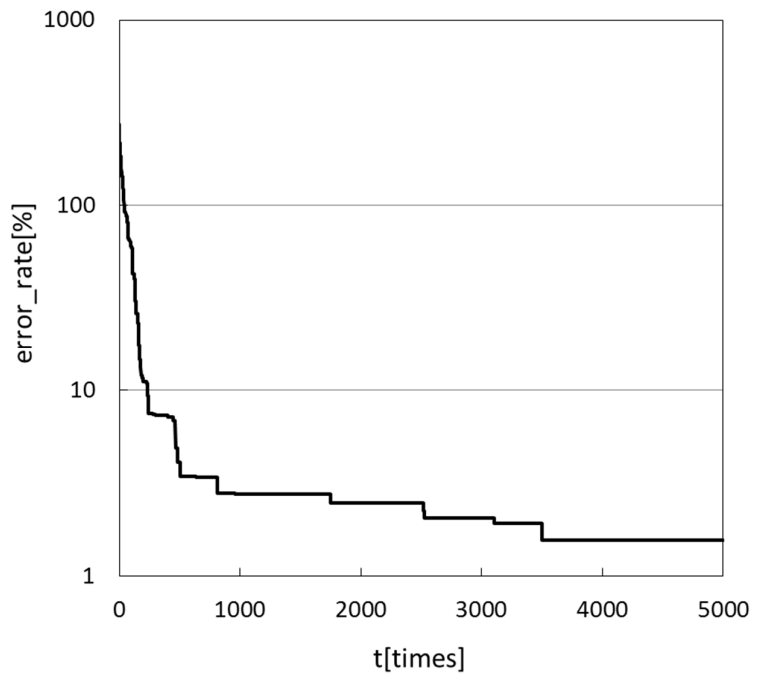

Fig. 7. error curve of GAVI.

result, the result of GAVI was better than GA. It was efficient that not only GA but also Infection applied in TSP. GAVI was valuable in convergence of the solution. We confirmed that VTE is efficient in TSP.

In future work, we would like to study the mechanism of Infection in detail. In this study, all number of element in Infection is depended on fixed probability. However changing fixed probability is to expected efficient. For example, large number of element is chosen frequent at first, while small number of element is chosen frequent latter. Convergence speed is fast at first, obtain solution prevent local minimum latter. Thus, we are expected to obtain a better solution in GAVI.

\section{ACKNOWLEDGMENT}

This work was partly supported by JSPS Grant-in-Aid for Young Scientists 23700269.

\section{REFERENCES}

[1] Little J, Murty K, Sweeney D, Karel C, “An algorithm for the traveling salesman problem,” Operations Research 11:972-89, 1963.

[2] Jean-Yves Potvin, "Genetic algorithms for the traveling salesman problem," Annals of Operations Research 63, pp.339-370, 1996.

[3] Kitano H ed., "Identeki Algorithm[Genetic Algorithm]," 1st ed. Sangyo Tosyo Kabushiki Kaisya, 1993.

[4] Fang G, Hongwei L, Qiang Z, Gang C, "A Genetic Algorithm with a Mixed Region Search for the Asymmetric Traveling Salesman Problem," Computers and Operations Research 30(5), 773-786, 2003.

[5] Naoshi N, Akinori K and Kunio K, "An Evolutionary Algorithm Based on the Virus Theory of Evolution," Information Processing Society of Japan Vol.40, No.5, 1999.

[6] Gao F, Liu H, Zhao Q, Cui G, "Virus-evolutionary particle swarm optimization algorithm,” vol 4222. Springer, Berlin, pp 156-165, 2006.

[7] Hideomi N, Takashi S and Takashi F, "Virus theory of evolution," Bulletin of Yamanashi Medical University Vol.3, pp.14-18, 1986.

[8] Zhaxybayeva O, Doolittle W F, "Lateral gene transfer," Curr. Biol. 21, R242-246 2011. 